\title{
Análisis de la producción científica latinoamericana sobre estrés docente $(2010$ - 2018)
}

\section{An analysis of Latin American Scientific Production on Teacher Stress (2010 - 2018)}

Carlos Augusto Luy-Montejo*

Universidad Tecnológica del Perú, Lima, Perú

ORCID: https://orcid.org/0000-0003-0824-7959

Julia Teves Quispe

Universidad Nacional Mayor de San Marcos, Lima, Perú

ORCID: https://orcid.org/0000-0002-7009-9937

Wilfredo Rojas Rivera

Universidad Nacional Daniel Alcides Carrión, Pasco, Perú

ORCID: https://orcid.org/0000-0002-8210-115X

Teresa Ramos Quispe

Universidad Nacional de San Agustín de Arequipa, Arequipa, Perú ORCID: http://orcid.org/0000-0003-4607-4745

Alan Daniel Lazo de la Vega Ramos

Universidad Nacional de San Agustín de Arequipa, Arequipa, Perú ORCID: https://orcid.org/0000-0003-2718-9985

Dennis Arias Chávez

Universidad Continental, Arequipa, Perú

ORCID: https://orcid.org/0000-0003-1500-8366

Dante Aguinaga-Villegas

Universidad Nacional Mayor de San Marcos, Lima, Perú ORCID: https://orcid.org/0000-0001-5166-0509

\section{Eric Gálvez-Suarez}

Universidad Nacional Mayor de San Marcos, Lima, Perú ORCID: https://orcid.org/0000-0002-7642-5019

Recibido 29-08-19 Revisado 15-09-19 Aprobado 01-10-18 En línea 02-10-19

*Correspondencia

Email: fracarlitos@gmail.com
Citar como:
Luy-Montejo, C., Teves, J., Rojas, W., Ramos, T., Lazo de la Vega, A., Arias, D., Aguinaga-Villegas, D., \& Gálvez-Suarez, E. (2019). Análisis de la producción científica latinoamericana sobre estrés docente (2010 - 2018). Propósitos y Representaciones, $\quad 7(3), \quad 01-11$ http://dx.doi.org/10.20511/pyr2019.v7n3.392 


\section{Resumen}

La presente investigación tuvo como objetivo evaluar la producción científica sobre estres docente. Es un estudio descriptivo transversal de los artículos publicados en revistas indizadas en la colección SciELO entre los años 2010 y 2018. Se analizó estimación de publicaciones por año, revistas, afiliaciones institucionales y temáticas de publicación. Se encontró que entre los años mencionados se han publicado 60 artículos sobre la temática, de los cuales los años de mayor producción fluctuan entre 2014 a 2018, las principales revistas de publicación sobre este tópico son: Actualidades Investigativas en Educación, Revista Salud Uninorte y Revista de Ciencias Médicas de Pinar del Río; además Brasil, Colombia y Cuba son los afiliaciones a instituciones de estos países con mayor cantidad de artículos publicaods. Podemos concluir que el estres docente como variable de investigación viene siendo estudiado de a poco en países latinoamericanos; sin embargo, es necesario seguir fortaleciendo estos estudios en demás bases de datos.

Palabras clave: Producción científica, Latinoamerica, Colección SciELO; Estrés docente.

\section{Summary}

The purpose of this research was to evaluate the scientific production on teacher stress. It is a cross-sectional descriptive study of articles published in journals indexed in the SciELO collection between 2010 and 2018. An estimate of publications per year, journals, institutional affiliations and publication topics was analyzed. It was found that between the aforementioned years, 60 articles on the subject have been published, out of which the years of greatest production fluctuate between 2014 and 2018. The main journals that published articles on this topic are: Research News in Education, Uninorte Health Magazine and the Magazine of Medical Sciences of Pinar del Río in Cuba. In addition, Brazil, Colombia and Cuba have the institutional affiliations with the highest number of published articles. We can conclude that teacher stress as a research variable has been studied gradually in Latin American countries; however, it is necessary to continue strengthening these studies in other databases.

Keywords: Scientific Production; Latin America; SciELO Collection; Teaching Stress.

\section{Introducción}

El trabajo es considerado como una actividad individual y colectiva del ser humano que requiere de una serie de características particulares como el tiempo, esfuerzo, compensaciones (Acosta \& Burgullos, 2014; Reynaga-Estrada, Arévalo Vázquez, Verdesoto Gáleas, Jiménez Ortega, Preciado Serrano, \& Morales Acosta, 2016). De esta manera, el trabajo involucra una serie de condiciones cognitivas, fisiológicas y biológicas que orientan hacia la prestación de un servicio o la producción de un bien, con una utilidad social: la satisfacción de una necesidad personal o de otras personas (Neffa, 2003).

El estrés relacionado con el trabajo se define como las reacciones emocionales, fisiológicas, cognitivas y del comportamiento a distintos aspectos adversos al entorno de la organización (Acosta \& Burgullos, 2014). Para el estrés se considera un problema de salud pública sin distinción de edad, sexo o nivel socioeconómico, con repercusiones en las funciones básicas del organismo (López \& López, 2014). Estudios realizados por la Unión Europea, sugieren que el estrés relacionado con el trabajo es el segundo problema de salud más común, afectando al 28\% de los trabajadores; y supone el 50\% o 60\% del total de los días laborales perdidos (Agencia Europea para la Seguridad y la Salud en el Trabajo, 2002). Otro dato importante lo menciona Navinés, Martín-Santos, Olivé, y Valdés, 2016 donde hace hincapié que hasta el 40\% de las personas mayores de 12 años reconocen haber experimentado estrés en algún momento de su vida cotidiana. El estrés podrá convertirse en cualquier respuesta a situaciones no específicas del cuerpo (Selye, 1974; Muñoz, Rumie, Torres, \& Villarroel, 2015). Las situaciones estresantes den generarse como resultado de diversos acontecimientos vitales, entre los que destacan los 
problemas laborales, las dificultades económicas y las amenazas a la salud (Navinés, MartínSantos, Olivé, \& Valdés, 2016). Ello implica que cualquier persona aprenda a asimilar y manejar y aceptar las limitaciones y aprenda a convivir afrontando situaciones de riesgo (López-Cruz, Hernández-Castañón, Mendoza-Zamora, Villarreal-Ríos, \& Gasca-Ramírez, 2016).

El estrés docente es visto como una de las temáticas que durante los últimos años ha llamado la atención e interés de muchos investigadores, de hecho, muchos estudios han señalado resultados entorno a que el docente es uno de los colectivos más vulnerables al desarrollo de estrés laboral y burnout (Villardefrancos, Santiago, Castro, Ache \& Otero-López, 2015). La búsqueda de una literatura científica ha permitido determinar las principales implicancias de los posibles focos de actuación a nivel preventivo y de intervención, lo que conlleva a indicar que existen determinantes exógenos al malestar laboral. El contexto educativo y los nuevos roles de los distintos agentes educativos hacen que el docente se sienta frustrado cuando descubre que no puede solucionar todas las problemáticas, superando dichas demandas sus posibilidades. Esta realidad genera un desgaste personal importante, que puede conducir al estrés crónico, al desgaste emocional e incluso al síndrome de burnout (Malander, 2016).

La producción científica de una variable atiende a los resultados de investigación e innovación y se ha convertido en una práctica común en áreas disciplinares. Su estudio se ha intensificado y sistematizado a partir de las últimas dos décadas (Piedra-Salomón, \& MartínezRodríguez, 2007). Las revistas científicas se han convertido en las principales vías de divulgación de resultados de investigación y de institucionalización social de la ciencia en la mayoría de los campos del conocimiento (Kreimer, 1998).

El objetivo del estudio fue describir el desarrollo de la producción científica latinoamericana sobre estrés docente, en la colección SciELO, lo que ayudará a conocer las fortalezas, y limitaciones de esta variable en el mundo académico y de investigación.

\section{Metodología}

Se realizó un estudio descriptivo transversal que consistió en la búsqueda, extracción y análisis de publicaciones científicas latinoamericanas enmarcados al estrés docente durante el periodo 2010-2018. La búsqueda fue realizada en la colección SciELO. Se realizó la búsqueda en el título, resumen y palabras clave empleando términos de búsqueda y añadiendo un filtro para el periodo estudiado. Se recuperaron 60 publicaciones las cuales conformaron la muestra para los resultados de este estudio

\section{Resultados}

Con respecto al número de publicaciones por año, se observa un aumento de publicaciones, manteniéndose un promedio de 4 a 5 artículos por año, y se evidencia, además, un aumento en las publicaciones entre los años 2014 a 2018, ver figura 1.

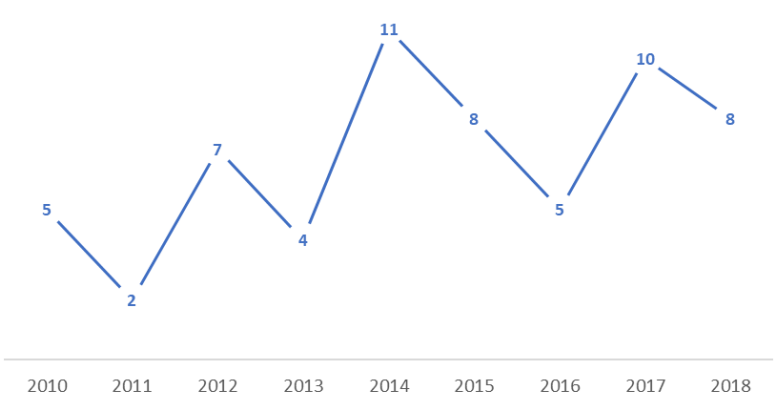

Figura 1. Estimación de las publicaciones por año. 
Los artículos analizados fueron publicados en 47 revistas. La Tabla 1 muestra las revistas donde se han publicado los artículos con referencia a la variable estrés docente. Se identifica que el 15\% de publicaciones corresponde a tres revistas con 3 artículos publicados cada una, mientras que el $61.7 \%$ involucra a publicaciones individuales en diferentes revistas (ver tabla 1).

Tabla 1.

Revistas donde se publican artículos científicos sobre estrés docente

\begin{tabular}{lccc}
\hline & $\mathrm{f}$ & $\%$ & $\begin{array}{c}\text { H Index } \\
\text { SJR }\end{array}$ \\
\hline Actualidades Investigativas en Educación & 3 & 5.0 & ND \\
\hline Revista Salud Uninorte & 3 & 5.0 & 9 \\
\hline Revista de Ciencias Médicas de Pinar del Río & 3 & 5.0 & ND \\
\hline Ciencia \& trabajo & 2 & 3.3 & ND \\
\hline Educação e Pesquisa & 2 & 3.3 & 13 \\
\hline Formación universitaria & 2 & 3.3 & 10 \\
\hline MEDISAN & 2 & 3.3 & ND \\
\hline Psicología Escolar e Educacional & 2 & 3.3 & 6 \\
\hline Revista de Investigacion Psicológica & 2 & 3.3 & ND \\
\hline Universidad y Salud & 2 & 3.3 & ND \\
\hline $\begin{array}{l}\text { Otras revistas con una sola publicación sobre } \\
\text { estrés docente }\end{array}$ & 37 & 61.7 & \\
\hline
\end{tabular}

En 60 artículos se pudo identificar a los autores con afiliación a instituciones latinoamericanas, destacando a Brasil como el país con autoría que publica mayormente sobre estrés docente, seguido de Colombia y Cuba (ver tabla 2).

Tabla 2.

Afiliación a instituciones de países latinoamericanos que han publicado artículos sobre estrés docente.

\begin{tabular}{lcc}
\hline & $\mathrm{f}$ & $\%$ \\
\hline Brasil & 15 & 25.0 \\
\hline Colombia & 13 & 21.7 \\
\hline Cuba & 8 & 13.3 \\
\hline México & 6 & 10.0 \\
\hline
\end{tabular}




\begin{tabular}{lcc}
\hline Chile & 5 & 8.3 \\
\hline Perú & 4 & 6.7 \\
\hline Costa Rica & 3 & 5.0 \\
\hline Bolivia & 2 & 3.3 \\
\hline Uruguay & 2 & 3.3 \\
\hline Venezuela & 2 & 3.3 \\
\hline
\end{tabular}

Según las áreas temáticas de las revistas incluidas en la colección SciELO, destaca la presencia de publicaciones de estrés docente en revistas con tópicos de ciencias de la salud, seguido de humanidades y ciencias sociales aplicadas (ver figura 2)

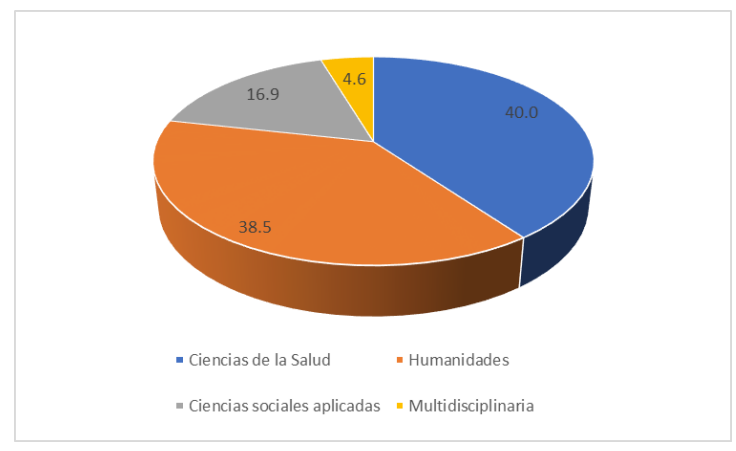

Figura 2. Temáticas de revistas de mayor incidencia en publicación sobre estrés docente

\section{Discusión}

El análisis de la producción latinoamericana sobre estrés docente se ha incrementado en los últimos años convirtiéndose en un referente del campo educativo. Resultados como las publicaciones en revistas de ciencias de la salud y humanidades nos llevan a mencionar el interés que existe en este tópico (Martínez, 2015). Además, los avances de producción conllevan a obtener una mayor visibilidad del estrés y su repercusión laboral, específicamente en el docente. Por ello, existe una creciente necesidad de seguir brindando recursos para paliar los efectos, llámese causa y/o consecuencias de esta variable.

Teniendo en cuenta la participación que evidencia el docente en el ámbito educativo, se sugiere que las instituciones desarrollen programas de prevención e intervención para aumentar la satisfacción laboral y la adquisición de capacidades que permitan adiestrar a estos agentes educativos en cómo enfrentar los desafíos de la labor docente, y de esta manera prevenir el desarrollo del estrés (Quass, 2006; Cantón \& Téllez, 2016).

Es importante mencionar que el avance científico en el estrés docente y la participación Latinoamericana sigue cumpliendo un rol central en el campo de las ciencias de la salud y ciencias sociales; sin embargo, los análisis de producción de esta variable aún son muy limitados, no existe un análisis general que implique la pertinencia de las casusas y efectos de este. El interés por las revistas donde se publican las investigaciones es un punto relevante en el conocimiento del estado de una disciplina y una muestra son los estudios en revistas temáticas (Urquidi \& Rodríguez, 2011). Pese a la importancia de este tipo de estudios, no se ha encontrado ningún estudio 
bibliométrico sobre estrés docente, por lo que el presente trabajo puede ser un punto de partida de nuevas investigaciones

\section{Referencias}

Agencia Europea para la Seguridad y la Salud en el Trabajo. FACTS. (2002). El estrés relacionado con el trabajo. http://agency.osha.eu.int

Acosta Contreras, M., \& Burguillos Peña, A. (2016). Estrés y burnout en profesores de primaria y secundaria de Huelva: las estrategias de afrontamiento como factor de protección. International Journal of Developmental and Educational Psychology. Revista INFAD de Psicología, 4(1), 303-310. doi: http://dx.doi.org/10.17060/ijodaep.2014.n1.v4.616

Cantón, I., \& Téllez, S. (2016). La satisfacción laboral y profesional de los profesores. Revista Lasallista de Investigación, 13(1), 214-226.

Kreimer, Pablo. (1998). Publicar y castigar. El papel como problema y la dinámica de los campos científicos. Revista de estudios sociales de la ciencia, 5(12), 51-73.

López, F., \& López M. (2014). Situaciones generadoras de estrés en los estudiantes de enfermería en las prácticas clínicas. Ciencia y enfermería, 17(2), 47-54. Recuperado de http://www.scielo.cl/scielo.php.

López-Cruz, V., Hernández-Castañón, M., Mendoza-Zamora, A., Villarreal-Ríos, E., \& GascaRamírez, M. (2016). Apoyo a estudiantes de enfermería en su primera práctica clínica ante situaciones de estrés. Revista de Enfermerí-a del Instituto Mexicano del Seguro Social, 24(3), 165-170. Recuperado de http://revistaenfermeria.imss.gob.mx/editorial/index.php/revista_enfermeria/article/view/ $179 / 283$

Malander, N. (2016). Síndrome de Burnout y Satisfacción Laboral en Docentes de Nivel Secundario. Ciencia \& trabajo, 18(57), 177-182. doi: https://dx.doi.org/10.4067/S071824492016000300177

Martínez, J. (2015). How secondary school teachers protect themselves from stress: burnout and coping strategies. Revista de Psicología del Trabajo y de las Organizaciones, 31(1), 1-9. https://dx.doi.org/10.1016/j.rpto.2015.02.001

Muñoz, C., \& Rumie, H., Torres, G., \& Villarroel, K. (2015). Impacto en la salud mental de la(del) enfermera(o) que otorga cuidados en situaciones estresanteS. Ciencia y enfermería, 21(1), 45-53. doi: https://dx.doi.org/10.4067/S0717-95532015000100005

Navinés, R., Martín-Santos, R., Olivé, V., \& Valdés, M. (2016). Estrés laboral: implicaciones para la salud física y mental. Medicina Clínica, 146(8), 359-366.

Neffa, J. C. (2003). El trabajo humano. Contribuciones al estudio de un valor que permanece. Buenos Aires: Lumen-Humanitas, Trabajo y Sociedad -CEILPIETTE/CONICET.

Piedra-Salomón, Y., \& Martínez-Rodríguez, A. (2007). Producción científica. Ciencias de la Información, 38(3), 33-38.

Quass, C. (2006). Diagnóstico de burnout y técnicas de afrontamiento al estrés en profesores universitarios de la Quinta Región de Chile. Psicoperspectivas. Individuo y Sociedad, 5(1), 65-75. doi: https://dx.doi.org/10.5027/psicoperspectivas-Vol5-Issue1-fulltext-35

Reynaga-Estrada, P., Arévalo Vázquez, E., Verdesoto Gáleas, Á., Jiménez Ortega, I., Preciado Serrano, M., \& Morales Acosta, J. (2016). Beneficios psicológicos de la actividad física en el trabajo de un centro educativo (Psychological benefits of physical activity in an educational center work). Retos, O(30), 203-206. Recuperado de https://recyt.fecyt.es/index.php/retos/article/view/50254

Selye H. (1974). The evolution of the stress concept. American Scientist, 61,692-99

Urquidi, L., \& Rodríguez, J. (2011). Estrés en profesorado universitario mexicano. Actualidades Investigativas en Educación, 10(2), 01-21. Recuperado de https://revistas.ucr.ac.cr/index.php/aie/article/view/10111

Villardefrancos, E., Santiago M., Castro, C., Ache, S., Otero-López, J. (2015). Estrés en profesores de enseñanza secundaria: un análisis desde el optimismo. European Journal of investigation in health, psychology and education, 2(3), 91-101. 